\title{
Reproductive History and Risk of Cognitive Impairment in Elderly Women: A Cross-Sectional Study in Eastern China
}

\author{
Fu-Dong $\mathrm{Li}^{\mathrm{a}, 1}$, Fan $\mathrm{He}^{\mathrm{a}, 1}$, Ting-Rui Chen ${ }^{\mathrm{b}, 1}$, Yuan-Yuan Xiao ${ }^{\mathrm{a}, \mathrm{c}}$, Shang-Tong Lin ${ }^{\mathrm{b}}$, Wei Shen ${ }^{\mathrm{a}}$, \\ Xin-Yi Wang ${ }^{\mathrm{a}}$, Yu-Jia Zhai ${ }^{\mathrm{a}}$, Xiao-Peng Shang ${ }^{\mathrm{a}}$ and Jun-Fen Lin ${ }^{\mathrm{a}, *}$ \\ ${ }^{a}$ Zhejiang Provincial Center for Disease Control and Prevention, Hangzhou, Zhejiang, China \\ ${ }^{\mathrm{b}}$ Cangnan Center for Disease Prevention and Control, Wenzhou, Zhejiang, China \\ ${ }^{\mathrm{c}}$ Kunming Medical University, Kunming, Yunnan, China
}

Accepted 4 August 2015

\begin{abstract}
.
Background: Epidemiological studies suggest that proxies of higher lifetime estrogen exposure are associated with better cognitive function in postmenopausal women, but this has not been found consistently.

Objective: To determine whether reproductive history, an important modifier of estrogen exposure across the lifetime, is associated with risk of cognitive impairment in postmenopausal women.

Methods: We analyzed the baseline data from Zhejiang Major Public Health Surveillance Program (ZPHS) including 4,796 postmenopausal women. Cognitive impairment was assessed through the application of Mini-Mental State Examination questionnaire. Logistic regression models, controlled for an extensive range of potential confounders, were generated to examine the associations between women's reproductive history and risk of cognitive impairment in their later life.

Results: The length of reproductive period was inversely associated with risk of cognitive impairment $(p=0.001)$. Odds ratio (OR) of cognitive impairment were 1.316 (95\% CI 1.095 1.582) for women with 5 or more times of full-term pregnancies, compared with those with 1 4 times of full-term pregnancies. Women without incomplete pregnancy had a significant higher risk of cognitive impairment ( $\mathrm{OR}=1.194,95 \%$ CI $1.000 \sim 1.429)$, compared with the reference ( $1 \sim 2$ times of incomplete pregnancies). Oral contraceptive use $(\mathrm{OR}=0.489,95 \%$ CI $0.263 \sim 0.910)$ and intrauterine device (IUD) use $(\mathrm{OR}=0.684,95 \%$ CI $0.575 \sim 0.815$ ) were associated with significantly reduced risk of cognitive impairment.

Conclusion: Our results indicated that shorter reproductive period, higher number of full-term pregnancies and no incomplete pregnancy history were associated with an increased risk of cognitive impairment. In contrast, oral contraceptive and IUD use corresponded to reduced risk of cognitive impairment.
\end{abstract}

Keywords: Cognitive impairment, estrogen, intrauterine device, pregnancies, reproductive history

\section{INTRODUCTION}

Diseases related to cognitive impairment, especially dementia and its most common cause "Alzheimer's disease" (AD), are becoming prominent health threat and bring enormous social burden in elders worldwide. It

\footnotetext{
${ }^{1}$ These authors contributed equally to this work.

${ }^{*}$ Correspondence to: Professor Jun-Fen Lin, Zhejiang Provincial Center for Disease Control and Prevention, 3399 Binsheng Road, Binjiang District, Hangzhou 310051, Zhejiang, China. Tel.: +86571 87115131; Fax: +86 571 87115147; E-mail: zjlinjunfen@ 163.com.
}

is estimated that more than 35.6 million people lived with dementia worldwide in 2010; this number will be doubled by 2030 , and tripled by 2050 [1]. Based on estimation, the prevalence of dementia and $\mathrm{AD}$ among individuals aged 65 years and older in China were around $5.14 \%$ and $3.21 \%$ respectively [2], and are increasing nationwide.

Extensive evidences showed that postmenopausal women carry an increased risk of developing $\mathrm{AD}$ than age-matched men [3-5], probably due to the marked reduction of estrogen level that occurs following the 
menopause. Animal and in vitro studies have identified that estrogen has several possible neuroprotective effects on the cognitive function, including inhibition of amyloid- $\beta$ formation [6], stimulation of cholinergic activity [7], protection against oxidative stress [8], increase of cerebral blood flow [9], etcetera. Nonetheless, controversy still exists in human studies.

Seeing that estrogen exposure may modify women's risk of cognitive impairment in later life, reproductive events, which influence the cumulative estrogen exposure across women's lifetime, were investigated in many epidemiological and clinical studies. The majority of studies supported that better cognitive performance in later life was associated with proxies of higher lifetime estrogen exposure, including longer reproductive period (years between menarche and menopause) and less parity [10-12]. On the contrary, others observed a negative or no association $[13,14]$. Moreover, to our knowledge, there is a vacancy in literature on the association between risk of cognitive impairment and number of incomplete pregnancies, which may result in different effect on estrogen levels in contrast to full-term pregnancy. In addition, only a few studies focusing on the association between women's reproductive history and risk of cognitive impairment have been conducted in Chinese populations, although previous studies revealed that estrogen concentration in Chinese women was generally lower than in Caucasian women in both pre and post menopause period $[15,16]$. Lastly, use of an intrauterine device (IUD), a form of longterm reversible contraception, induces a number of immunological and biochemical changes in the uterine environment and possibly affects the women's hormonal concentration, and it received little attention previously in this field.

In this study, we utilized the baseline data from a large population-based cohort study to explore the associations between reproductive history (including incomplete pregnancy especially) and cognitive impairment among elderly women in eastern China, and discussed IUD users' risk of cognitive impairment.

\section{MATERIALS AND METHODS}

\section{Study design}

The baseline data is from Zhejiang Major Public Health Surveillance Program (ZPHS), a community based cohort study focusing on aging and health among elderly in Zhejiang province, China. It was conducted by Zhejiang Provincial Center for Disease Control and
Prevention, and the baseline survey was finished in 2014. Seven counties were randomly selected from 90 counties in Zhejiang province. At least 1,500 permanent residents in each county aged 60 years and older were randomly selected for participation. Finally 9,411 $(89.6 \%)$ valid questionnaires were obtained. During the baseline survey, ZPHS performed questionnairebased interview, physical examinations and laboratory tests for each participant. ZPHS protocol was approved by the Ethics Committee of Zhejiang Provincial Center for Disease Control and Prevention, and written consents were obtained from all participants prior to the research.

\section{Participant selection}

We selected postmenopausal women from the database stated above. Female participants who answered "yes" to the question "Do you have menopause already?" were recognized as eligible study participants. Data completeness was checked subsequently, and observations with incomplete information in cognitive function and reproduction characteristics were deleted.

\section{Cognitive assessment}

Cognitive functioning was assessed using the Chinese version of the Mini-Mental State Examination (MMSE), which includes 30 items. The maximum score is 30 points, with higher MMSE scores indicating better cognitive function. The widely accepted cut-off point to define cognitive impairment in China (MMSE Chinese Standard, MCS) is education-specific [17]: 17/18 for illiteracy, 20/21 for people with primary education level, 24/25 for people with higher than primary education level. We defined cognitive impairment according to this standard.

\section{Reproductive characteristics}

Factors that were possibly related to endogenous or exogenous estrogen exposure across the lifetime were collected in the reproductive history section within the questionnaire: age at menarche, age at menopause, regularity of menstrual cycle ("regular" and "irregular"), length of menstrual cycle, number of full-term pregnancies, number of incomplete pregnancies, oral contraceptive use ("ever" and "never"), IUD use ("ever" and "never"). Reproductive period, an indicator of endogenous estrogen exposure, was calculated as age at menopause minus age at menarche. 


\section{Other variables}

Based on findings reported in the literature, variables described below were considered as potential confounders and were included in our analysis: age, race ("Han ethnicity" and "minority"), education level ("lower than primary", "primary", "junior middle", "senior middle" and "college and above"), marital status ("single", "married" and "divorced/widowed"), economic status ("rich", "median" and "poor"), smoking ("never", "past" and "current"), alcohol drinking ("never", "past" and "current"), exercise ("yes" and "no"), hypertension ("yes" and "no"), diabetes ("yes" and "no"), coronary heart disease ("yes" and "no"), and depressive symptom ("yes" and "no"). Economic status was self-evaluated and reported by participants. Depressive symptom was determined using the Patient Health Questionnaire9 scale (PHQ-9) and postmenopausal women who scored 5 or above were defined as depressed [18].

\section{Statistical analysis}

Descriptive statistics were applied to illustrate the general characteristics and reproductive characteristics of included postmenopausal women. Crude age-specific prevalence of cognitive impairment were calculated according to number of full-term pregnancies and number of incomplete pregnancies. The associations between general characteristics and cognitive function were examined by $t$-test or Chi-square test or Kruskal-Wallis test as appropriate to variables. The general characteristics which were associated with poor cognitive performance at the $10 \%$ significance level $(p<0.1)$ were considered as potential confounders. Preliminary logistic regression model (model 1) was generated by including all identified potential confounders, to screen possible associated factors of reproductive characteristics and risk of cognitive impairment one by one, then, independents with $p$ values $<0.1$ were included into further adjusted logistic regression model (model 2) to estimate the adjusted ORs. Interactions between independents were checked simultaneously in model 2, with the inclusion criterion defined as $p<0.05$.

All statistical analyses were performed by SPSS (version 20.0, SPSS Inc.), and the significance level was at two-tailed probability $<0.05$.

\section{RESULTS}

\section{General and reproductive characteristics of participants}

By using participants selection criteria mentioned above, we initially identified 4,805 postmenopausal women from an accumulation of 9,411 participants. After checking for data completeness on MMSE scores, 4,796 postmenopausal women were included in final analysis. The general characteristics of included postmenopausal women are presented in Table 1.

Among the 4796 postmenopausal women, 919 were indicated as cognitive impairment by MMSE, and the prevalence of cognitive impairment was $19.2 \%$ (95\% CI 18.1 20.3). The mean age of postmenopausal women was 69.8 years, with a higher mean age (75.0 years) in women suffering cognitive impairment $(p<0.001)$. Besides age, the majority of variables were significantly associated with cognitive function, such as race, education, marital status, economic status, smoking, alcohol drinking, exercise, hypertension, and depression, so these variables were considered as potential confounders and were adjusted in further analysis. Meanwhile, the distributions of diabetes and coronary heart disease showed no difference between women with and without cognitive impairment.

Descriptive statistics for reproductive characteristics of included postmenopausal women were shown in Table 2. The postmenopausal women in this study started menopause on average 32.5 years after menarche, with an average of every 29.3 days to complete a menstrual cycle during reproductive period. Only $6 \%$ reported irregular menstrual cycle. Over three quarters of all women had $1 \sim 4$ times of full-term pregnancies, and 3,185 (66.5\%) did not experience miscarriage or abortion. 154 $(3.2 \%)$ and $2,470(51.6 \%)$ women reported ever use of oral contraceptive and an IUD, respectively.

\section{Association between reproductive characteristics and cognitive function}

We explored the associated factors of cognitive impairment by logistic regression, controlled for all potential confounders identified. In the preliminary model, reproductive period, number of full-term pregnancies, number of incomplete pregnancies, oral contraceptive use, and IUD use were significantly associated with cognitive impairment. After adjusting for 
Table 1

General characteristics of 4796 included postmenopausal women

\begin{tabular}{|c|c|c|c|c|}
\hline \multirow[t]{2}{*}{ Characteristics } & \multirow[t]{2}{*}{ Overall $(n=4,796)$} & \multicolumn{2}{|c|}{ Cognitive impairment } & \multirow[t]{2}{*}{$\overline{p \text {-value }}$} \\
\hline & & Yes $(n=919)$ & No $(n=3,877)$ & \\
\hline Age (years, mean, SD) & $69.8(7.7)$ & $75.0(8.6)$ & $68.5(6.9)$ & $<0.001$ \\
\hline Race $(n, \%)$ & & & & $<0.001$ \\
\hline Han ethnicity & $4624(96.4)$ & $850(92.5)$ & $3774(97.3)$ & \\
\hline Minority & $172(3.6)$ & $69(7.5)$ & $103(2.7)$ & \\
\hline Education $(n, \%)$ & & & & $<0.001$ \\
\hline$<$ Primary & $3144(65.5)$ & 719 (78.2) & $2425(62.5)$ & \\
\hline Primary & $1531(31.9)$ & $184(20.0)$ & $1347(34.8)$ & \\
\hline Junior middle & $100(2.1)$ & $10(1.1)$ & $90(2.3)$ & \\
\hline Senior middle & $19(0.4)$ & $4(0.4)$ & $15(0.4)$ & \\
\hline$\geq$ College & $2(0.0)$ & $2(0.2)$ & $0(0.0)$ & \\
\hline Marital status $(n, \%)$ & & & & $<0.001$ \\
\hline Single & $19(0.4)$ & $4(0.4)$ & $15(0.4)$ & \\
\hline Married & $3221(67.2)$ & $470(51.1)$ & $2751(71.0)$ & \\
\hline Divorced/widowed & $1551(32.3)$ & $445(48.4)$ & $1106(28.5)$ & \\
\hline Economic status $(n, \%)$ & & & & $<0.001$ \\
\hline Rich & $431(9.0)$ & $53(5.8)$ & $378(9.7)$ & \\
\hline Median & $3773(78.7)$ & $676(73.6)$ & 3097 (79.9) & \\
\hline Poor & $592(12.3)$ & $190(20.7)$ & $402(10.4)$ & \\
\hline Smoking $(n, \%)$ & & & & 0.001 \\
\hline Never & $4686(97.7)$ & $883(96.1)$ & $3803(98.1)$ & \\
\hline Past & $55(1.1)$ & $19(2.1)$ & $36(0.9)$ & \\
\hline Current & $55(1.1)$ & $17(1.8)$ & $38(1.0)$ & \\
\hline Alcohol drinking $(n, \%)$ & & & & 0.033 \\
\hline Never & $4234(88.3)$ & $796(86.6)$ & $3438(88.7)$ & \\
\hline Past & $185(3.9)$ & $49(5.3)$ & $136(3.5)$ & \\
\hline Current & $377(7.9)$ & $74(8.1)$ & $303(7.8)$ & \\
\hline Exercise (yes, $n, \%$ ) & $914(19.1)$ & $129(14.0)$ & $785(20.2)$ & $<0.001$ \\
\hline Hypertension (yes, $n, \%$ ) & $2448(46.9)$ & $472(51.4)$ & $1776(45.8)$ & 0.002 \\
\hline Diabetes (yes, $n, \%$ ) & $508(10.6)$ & $101(11.0)$ & $407(10.5)$ & 0.663 \\
\hline $\mathrm{CHD}(\mathrm{yes}, n, \%)$ & $162(3.4)$ & $39(4.2)$ & $123(3.2)$ & 0.106 \\
\hline Depressive symptom (yes, $n, \%$ ) & $612(12.8)$ & $197(21.4)$ & $415(10.7)$ & $<0.001$ \\
\hline
\end{tabular}

$\mathrm{SD}$, standard deviation; CHD, coronary heart disease.

Table 2

Reproductive characteristics among 4796 postmenopausal women

\begin{tabular}{|c|c|c|c|}
\hline \multirow[t]{2}{*}{ Reproductive characteristics } & \multirow[t]{2}{*}{ Overall $(n=4,796)$} & \multicolumn{2}{|c|}{ Cognitive impairment } \\
\hline & & Yes $(n=919)$ & No $(n=3,877)$ \\
\hline Age at menarche (years, mean, SD) & $16.8(1.7)$ & $17.1(1.7)$ & $16.8(1.7)$ \\
\hline Age at menopause (years, mean, SD) & $49.3(4.2)$ & $48.8(4.3)$ & $49.4(4.2)$ \\
\hline Reproductive period (years, mean, SD) & $32.5(4.7)$ & $31.8(4.6)$ & $32.7(4.6)$ \\
\hline \multicolumn{4}{|l|}{ Regularity of menstrual cycle $(n, \%)$} \\
\hline Regular & $4505(94.0)$ & $859(93.6)$ & $3646(94.0)$ \\
\hline Irregular & $290(6.0)$ & $59(6.4)$ & $231(6.0)$ \\
\hline Length of menstrual cycle (days, mean, SD) ${ }^{\mathrm{a}}$ & $29.3(2.4)$ & $29.4(2.0)$ & $29.3(2.4)$ \\
\hline \multicolumn{4}{|l|}{ Number of full-term pregnancies $(n, \%)$} \\
\hline 0 & $58(1.2)$ & $17(1.9)$ & $41(1.1)$ \\
\hline $1-4$ & $3651(76.2)$ & $534(58.2)$ & $3117(80.5)$ \\
\hline$\geq 5$ & $1080(22.6)$ & $366(39.9)$ & $714(18.4)$ \\
\hline \multicolumn{4}{|l|}{ Number of incomplete pregnancies $(n, \%)$} \\
\hline 0 & $3185(66.5)$ & $644(70.2)$ & $2541(65.6)$ \\
\hline $1-2$ & $1473(30.8)$ & $240(26.2)$ & $1233(31.8)$ \\
\hline$\geq 3$ & $131(2.7)$ & $33(3.6)$ & $98(2.5)$ \\
\hline \multicolumn{4}{|l|}{ Oral contraceptives use $(n, \%)$} \\
\hline Never & $4622(96.8)$ & $906(98.7)$ & $3716(96.3)$ \\
\hline Ever & $154(3.2)$ & $12(1.3)$ & $142(3.7)$ \\
\hline \multicolumn{4}{|l|}{ IUD use $(n, \%)$} \\
\hline Never & $2319(48.4)$ & $624(68.0)$ & $1695(43.8)$ \\
\hline Ever & $2470(51.6)$ & $293(32.0)$ & $2177(56.2)$ \\
\hline
\end{tabular}

SD, standard deviation; IUD, intrauterine device. Data for the following are missing: 17 for age at menarche; 17 for age at menopause; 17 for reproductive period; 1 for regularity of menstrual cycle; 47 for length of menstrual cycle; 7 for number of full-term pregnancies; 7 for number

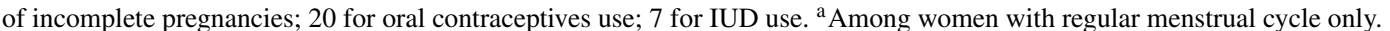


chosen independents, these associations remained significant in further logistic regression model (Table 3 ).

Reproductive period was used as a main proxy measure for endogenous estrogen exposure and was defined as the length of time between age at menarche and menopause. Our study found that longer reproductive period was related to a lower risk of cognitive impairment $(\mathrm{OR}=0.972$, 95\% CI 0.956 0.988, $p=0.001$ ), and this result was statistically significant after adjustment for other reproductive characteristics $(\mathrm{OR}=0.974,95 \%$ CI 0.958, 0.990, $p=0.001)$. It indicated that each additional reproductive year corresponded to a $3 \%$ reduction in risk of cognitive impairment. Neither the regularity of menstrual cycle nor the length of menstrual cycle, were associated with cognitive impairment.

Estrogen exposure is expected to be correlated with the number of pregnancies, including full-term and incomplete pregnancies. In our study, we tested both the relationship between number of full-term pregnancies, number of incomplete pregnancies, and cognitive impairment. Compared with women with 1 4 times of full-term pregnancies, a $30 \%$ increase in risk of cognitive impairment was observed in women with 5 or more times of full-term pregnancies, while the risk of cognitive impairment was non-significant in nulliparity. The risk of cognitive impairment in women without incomplete pregnancy was higher than women with $1 \sim 2$ times of incomplete pregnancies $(\mathrm{OR}=1.219,95 \%$ CI $1.002 \sim 1.452$, $p=0.027$ ), and the result was still statistically significant after adjusting for other additional reproductive characteristics $(\mathrm{OR}=1.194,95 \%$ CI $1.000 \sim 1.429$, $P=0.049$ ). Nevertheless, women with 3 or more times of incomplete pregnancies did not have a significant increased risk of cognitive impairment compared with women with fewer times of incomplete pregnancies. Figures 1 and 2 showed crude age-specific prevalence of cognitive impairment by number of full-term pregnancies and number of incomplete pregnancies, respectively.

We observed a statistically significant, almost a 50\% reduction in risk of cognitive impairment in women who had ever used oral contraceptives $(\mathrm{OR}=0.482$, $95 \%$ CI $0.261 \sim 0.892, p=0.020$ ), and a similar protective effect was observed in women who had ever used an IUD (OR $=0.669$, 95\% CI $0.563 \sim 0.794$, $p<0.001$ ), after adjusting for potential confounders. These associations were relatively steady in further logistic regression, which controlled for other reproductive characteristics.

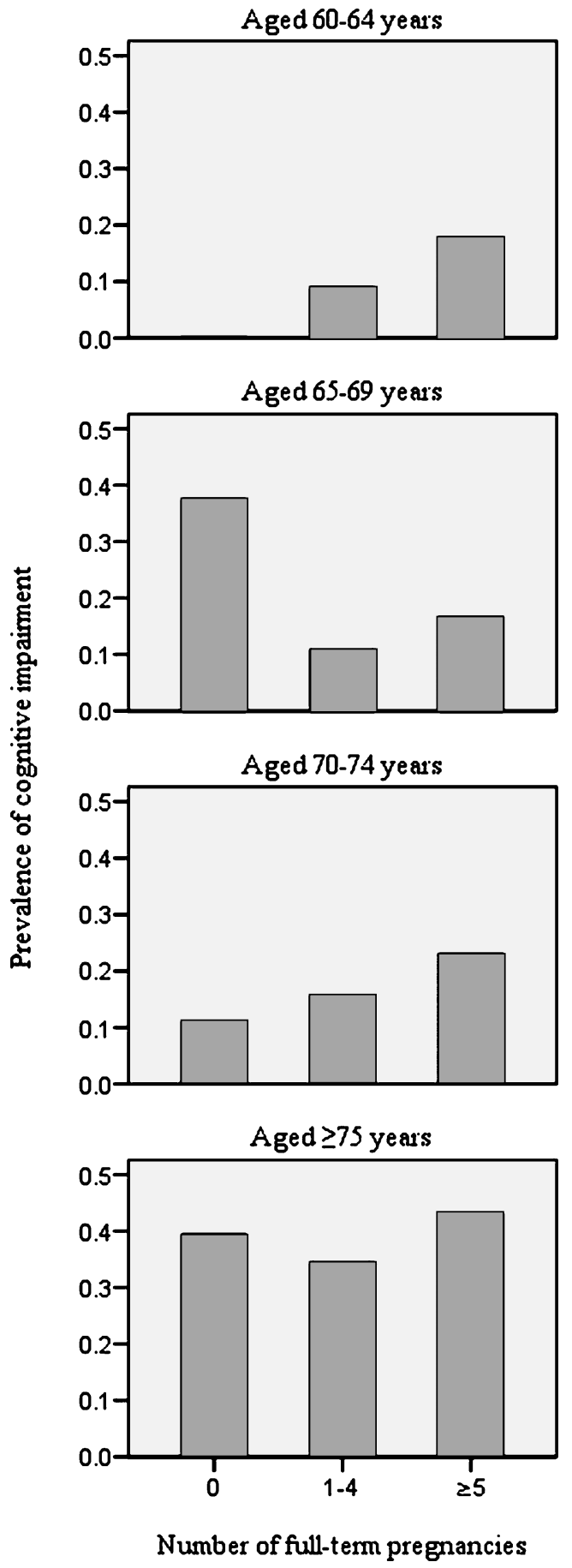

Fig. 1. Crude age-specific prevalence of cognitive impairment by number of full-term pregnancies.

\section{DISCUSSION}

In this study, we explored the relationship between reproductive characteristics and cognitive impairment. 


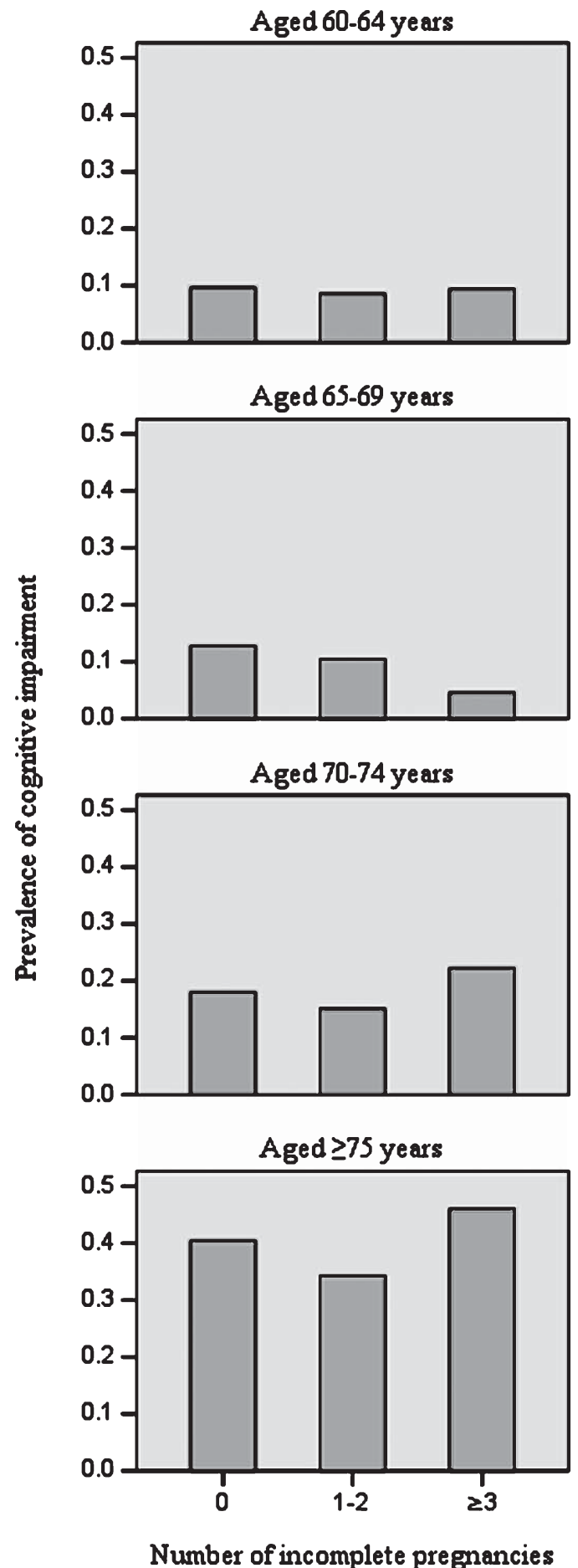

Fig. 2. Crude age-specific prevalence of cognitive impairment by number of incomplete pregnancies.

The results of our study suggested that shorter reproductive period, higher number of full-term pregnancies, and no incomplete pregnancy history were significantly associated with an increased risk of cognitive impairment, whereas oral contraceptives use and IUD use contributed to the beneficial effect on cognitive function.

Estrogen exposure may alter women's risk of cognitive impairment. Women differ in their level of cumulative estrogen exposure due to variances in reproductive history and use of estrogen containing therapies. Endogenous estrogen exposure is highest during a woman's reproductive life. An increasing number of reproductive years, resulting from a younger age at menarche and/or an older age at menopause, indicates a higher lifetime endogenous estrogen exposure [19]. We found that a longer reproductive period was associated with a significantly better cognitive function (MMSE), in agreement with the majority of previous studies [10-12]. Other studies [20-22] also reported the similar effect of reproductive period on the risk of dementia and AD, but not all studies [13, $23,24]$. Our finding supported the protective effect of estrogen exposure on cognitive impairment.

Although the length of reproductive period is associated with women's lifetime estrogen exposure, reproductive activity during this time also affects endogenous estrogen exposure. Evidences demonstrated that the level of estrogen varies differently during and after pregnancy $[25,26]$. Most previous studies only investigated the variables of number of pregnancies and number of children. Considering that there is no biological evidence that incomplete pregnancy produces the equivalent effect in estrogen levels as full-term pregnancy [20], we explored both fullterm pregnancy and incomplete pregnancy with their effects on cognitive function. Our results suggested that full-term pregnancy and incomplete pregnancy have different effects on cognitive function. We found that women with 5 or more times of full-term pregnancies had worse cognitive function, compared with those who had fewer times of full-term pregnancies. Previous studies generally supported that despite the high levels of estrogen during pregnancy, women with more parity were associated with the higher risk of cognitive impairment [10-12, 27] and AD [28-30], due to lower circulating estrogen over lifetime than women with fewer parity or nulliparity [25, 31]. In contrast, our results is the first to find that women with 3 or more times of incomplete pregnancies were not associated with the decline in cognitive performance compared with women who had fewer times of incomplete pregnancies. Moreover, women without incomplete pregnancy had a higher risk of cognitive impairment compared with the same reference, after controlling for general and other reproductive charac- 
Table 3

Association between reproductive characteristics and cognitive impairment

\begin{tabular}{|c|c|c|c|c|}
\hline \multirow[t]{2}{*}{ Reproductive characteristics } & \multicolumn{2}{|c|}{ Model $1^{\mathrm{a}}$} & \multicolumn{2}{|c|}{ Model $2^{\mathrm{b}, \mathrm{c}}$} \\
\hline & OR $(95 \% \mathrm{CI})$ & $p$-value & OR $(95 \% \mathrm{CI})$ & $p$-value \\
\hline Reproductive period & $0.972(0.956,0.988)$ & 0.001 & $0.974(0.958,0.990)$ & 0.001 \\
\hline Regularity of menstrual cycle (regular) & $0.889(0.644,1.227)$ & 0.474 & & \\
\hline Length of menstrual cycle ${ }^{\mathrm{d}}$ & $1.008(0.978,1.040)$ & 0.595 & & \\
\hline \multicolumn{5}{|l|}{ Number of full-term pregnancies } \\
\hline 0 & $1.026(0.543,1.939)$ & 0.937 & $0.816(0.434,1.536)$ & 0.529 \\
\hline $1-4$ & 1.000 (reference) & - & 1.000 (reference) & - \\
\hline$\geq 5$ & $1.360(1.133,1.632)$ & 0.001 & $1.316(1.095,1.582)$ & 0.003 \\
\hline \multicolumn{5}{|l|}{ Number of incomplete pregnancies } \\
\hline 0 & $1.219(1.002,1.452)$ & 0.027 & $1.194(1.000,1.429)$ & 0.049 \\
\hline $1-2$ & 1.000 (reference) & - & 1.000 (reference) & - \\
\hline$\geq 3$ & $1.343(0.855,2.110)$ & 0.201 & $1.274(0.808,2.009)$ & 0.297 \\
\hline Oral contraceptives use (ever) & $0.482(0.261,0.892)$ & 0.020 & $0.489(0.263,0.910)$ & 0.024 \\
\hline IUD use (ever) & $0.669(0.563,0.794)$ & $<0.001$ & $0.684(0.575,0.815)$ & $<0.001$ \\
\hline
\end{tabular}

OR, odds ratio; CI, confidence interval; IUD, intrauterine device. Sample size of each analysis in model 1 when following variables were screened: 4,779 for reproductive period; 4,795 for regularity of menstrual cycle; 4458 for length of menstrual cycle; 4789 for number of fullterm pregnancies; 4,789 for number of incomplete pregnancies; 4776 for Oral contraceptives use; 4,789 for IUD use. Sample size of model 2 was 4751. It was due to the missing values. ${ }^{a}$ Adjusted for potential confounders (age, race, education, marital status, economic status, smoking, alcohol drinking, exercise, hypertension and depressive symptom). ${ }^{b}$ Reproductive characteristics with $p$ values $<0.1$ in model 1 are included into model 2, and all potential confounders (same as model 1) are also adjusted. ${ }^{\mathrm{c}}$ No significant interactions were found between independents in model 2. ${ }^{\mathrm{d}}$ Among women with regular menstrual cycle only.

teristics (especially number of full-term pregnancies). Speculative explanation is that, women experience high levels of estrogen during pregnancy (no matter complete or incomplete pregnancy); however, incomplete pregnancy has weaker effect on longer-term reduction of estrogen levels than full-term pregnancy.

Besides endogenous estrogen exposure, exogenous estrogen use and related factors may also modify the risk of cognitive impairment and the incidence of $\mathrm{AD}$ [32]. Estrogen is one of major ingredients in oral contraceptive. Our finding of a protective effect of oral contraceptive use on cognitive function is consistent with some earlier population-based studies [12, 33], whereas no significant association was also identified $[13,34]$. Another study [27] found that oral contraceptive use was related to better cognitive performance only in univariate model. Mifepristone, a kind of emergency contraceptive, has been reported to be effective in treatment for $\mathrm{AD}$ in a number of clinical studies [35-37]. It is important to note that the particular type and duration of oral contraceptive used by participants were not collected in our study and also not reported by those epidemiological studies afore mentioned, and it is highly possible that different oral contraceptive may differ in their effect on cognitive function. This may help to explain some of the controversy with regard to the role of oral contraceptive use in protection of cognitive impairment and against AD.

The IUD is the most widely used single method of contraception in China and worldwide [38, 39]. Our study found that ever use of an IUD was inversely related to the risk of cognitive impairment. This potential association between IUD use and cognitive impairment, which was not assessed in previous literatures, needs to be confirmed in future study. Most women with IUD use $(98.5 \%)$ in our study practiced intrauterine contraception before 1990s, when inert IUDs and copper IUDs were most used among all types of IUDs in China [40]. Clinical studies suggested that inert IUD and copper IUD users had increased concentration of estrogen receptors [41] or higher levels of estrogen [42-46]. It is likely that IUD use protects the cognitive function through changing the balance of hormone receptors or hormone levels in the uterus. However, some other studies presented different evidence [47-50]. Therefore, it is unclear whether use of an IUD will lower the risk of cognitive impairment through hormonal mechanisms, an issue worth further study.

There are several methodological issues and limitations. First, some recall bias might exist considering the self-report essence of retrospective information, particularly concerning lifetime estrogen exposure. Although reproductive characteristics were events that occurred long before for recruitment of subjects in this study, research suggested that up to $90 \%$ of normal women could accurately report their age at menarche within a 1-year error margin [51]. Unfortunately, if the error rate of recalling retrospective information was higher in those women with more severe forms 
of cognitive impairment, it might have strengthened or weakened the association between reproductive characteristics and cognitive impairment. Despite this, the findings of this study did fit with predictions based on the effects of estrogens on cognitive function, and provided clues for further research. Secondly, there were no detailed information on specific type and duration of oral contraceptive and IUD, as well as the information about estrogen replacement therapy (ERT) use. The data of ERT use was actually collected through the questionnaires. However, only $18(0.4 \%)$ of 4,796 women reported ever use of ERT, while most of them experienced perimenopause before year 2000. Unlike western countries, ERT is gradually accepted and used in recent years in China. Even in urban areas, there are still a lot of menopause women without receiving ERT nowadays. These may explain the low rate of ERT use in our study. We could not excluded reporting bias of ERT use as well. Because of the small number of ERT users, we think it would not substantially influence the results of our study. Finally, we did not take genetic factors into consideration, such as apolipoprotein $\mathrm{E}$ (APOE) carrier status.

Despite these limitations, this study has a number of strengths. 1) Our study was based on a large community-based sample of postmenopausal women, and the results are relatively robust and are potentially correlated with the broader population of elderly women in Zhejiang province. 2) A wide range of potential confounders was taken into account in our analyses, and adjusted ORs were estimated in logistic model to control other reproductive characteristics. 3) Some important new findings were made in this study, including the relationship between the number of incomplete pregnancies, IUD use, and risk of cognitive impairment. 4) The limited number of studies based on Chinese population makes our study a valuable extension on previous work.

\section{ACKNOWLEDGMENTS}

We wish to thank the investigators from 7 selected counties for their investigation and data collection, and Yan-Jin Yu (Liuxia Family Planning Service Station) for her kind help and suggestions with this manuscript.

Authors' disclosures available online (http://jalz.com/manuscript-disclosures/15-0444r1).

\section{REFERENCES}

[1] Prince M, Bryce R, Albanese E, Wimo A, Ribeiro W, Ferri CP (2013) The global prevalence of dementia: A systematic review and metaanalysis. Alzheimers Dement 9, 63-75.e2.
[2] Jia J, Wang F, Wei C, Zhou A, Jia X, Li F, Tang M, Chu L, Zhou Y, Zhou C, Cui Y, Wang Q, Wang W, Yin P, Hu N, Zuo X, Song H, Qin W, Wu L, Li D, Jia L, Song J, Han Y, Xing Y, Yang P, Li Y, Qiao Y, Tang Y, Lv J, Dong X (2014) The prevalence of dementia in urban and rural areas of China. Alzheimers Dement 10, 1-9.

[3] The Italian Longitudinal Study On Aging Working, Group (1997) Prevalence of chronic diseases in older Italians: Comparing self-reported and clinical diagnoses. Int J Epidemiol 26, 995-1002.

[4] Birge SJ (1997) The role of estrogen in the treatment and prevention of dementia: Introduction. Am J Med 103, 1S-2S.

[5] Li R, Singh M (2014) Sex differences in cognitive impairment and Alzheimer's disease. Front Neuroendocrinol 35, 385-403.

[6] Amtul Z, Wang L, Westaway D, Rozmahel RF (2010) Neuroprotective mechanism conferred by 17 beta-estradiol on the biochemical basis of Alzheimer's disease. Neuroscience 169, 781-786.

[7] Luine VN (1985) Estradiol increases choline acetyltransferase activity in specific basal forebrain nuclei and projection areas of female rats. Exp Neurol 89, 484-490.

[8] Behl C, Widmann M, Trapp T, Holsboer F (1995) 17-beta estradiol protects neurons from oxidative stress-induced cell death in vitro. Biochem Biophys Res Commun 216, 473-482.

[9] Gibbs RB, Aggarwal P (1998) Estrogen and basal forebrain cholinergic neurons: Implications for brain aging and Alzheimer's disease-related cognitive decline. Horm Behav 34, 98-111.

[10] Rasgon NL, Magnusson C, Johansson AL, Pedersen NL, Elman S, Gatz M (2005) Endogenous and exogenous hormone exposure and risk of cognitive impairment in Swedish twins: A preliminary study. Psychoneuroendocrinology 30, 558-567.

[11] Heys M, Jiang C, Cheng KK, Zhang W, Au YS, Lam TH, Leung GM, Schooling CM (2011) Life long endogenous estrogen exposure and later adulthood cognitive function in a population of naturally postmenopausal women from Southern China: The Guangzhou Biobank Cohort Study. Psychoneuroendocrinology 36, 864-873.

[12] Low LF, Anstey KJ, Jorm AF, Rodgers B, Christensen H (2005) Reproductive period and cognitive function in a representative sample of naturally postmenopausal women aged 60-64 years. Climacteric 8, 380-389.

[13] Ryan J, Carriere I, Scali J, Ritchie K, Ancelin ML (2009) Lifetime estrogen exposure and cognitive functioning in later life. Psychoneuroendocrinology 34, 287-298.

[14] Henderson VW, Guthrie JR, Dudley EC, Burger HG, Dennerstein L (2003) Estrogen exposures and memory at midlife: A population-based study of women. Neurology 60, 1369-1371.

[15] Key TJ, Chen J, Wang DY, Pike MC, Boreham J (1990) Sex hormones in women in rural China and in Britain. Br J Cancer 62, 631-636.

[16] Bernstein L, Yuan JM, Ross RK, Pike MC, Hanich R, Lobo R, Stanczyk F, Gao YT, Henderson BE (1990) Serum Hormone Levels in premenopausal Chinese women in Shanghai and white women in Los Angeles: Results from two breast cancer case-control studies. Cancer Causes Control 1, 51-58.

[17] Wang Y (2005) The Rating Scales for Neurology, China Friendship Publishing Company, Beijing.

[18] Kroenke K, Spitzer RL, Williams JB (2001) The PHQ-9: Validity of a brief depression severity measure. J Gen Intern Med 16, 606-613.

[19] Clavel-Chapelon F (2002) Cumulative number of menstrual cycles and breast cancer risk: Results from the E3N cohort study of French women. Cancer Causes Control 13, 831-838. 
[20] Fox M, Berzuini C, Knapp LA (2013) Cumulative estrogen exposure, number of menstrual cycles, and Alzheimer's risk in a cohort of British women. Psychoneuroendocrinology 38, 2973-2982.

[21] Kim JM, Stewart R, Shin IS, Yoon JS (2003) Limb length and dementia in an older Korean population. J Neurol Neurosurg Psychiatry 74, 427-432.

[22] Hong X, Zhang X, Li H (2001) A case-control study of endogenous estrogen and risk of Alzheimer's disease. Zhonghua Liu Xing Bing Xue Za Zhi 22, 379-382.

[23] Colucci M, Cammarata S, Assini A, Croce R, Clerici F, Novello C, Mazzella L, Dagnino N, Mariani C, Tanganelli P (2006) The number of pregnancies is a risk factor for Alzheimer's disease. Eur J Neurol 13, 1374-1377.

[24] Geerlings MI, Ruitenberg A, Witteman JC, van Swieten JC, Hofman A, van Duijn CM, Breteler MM, Launer LJ (2001) Reproductive period and risk of dementia in postmenopausal women. JAMA 285, 1475-1481.

[25] Bernstein L, Pike MC, Ross RK, Judd HL, Brown JB, Henderson BE (1985) Estrogen and sex hormone-binding globulin levels in nulliparous and parous women. J Natl Cancer Inst 74, 741-745.

[26] Trichopoulos D, Cole P, Brown JB, Goldman MB, MacMahon B (1980) Estrogen profiles of primiparous and nulliparous women in Athens, Greece. J Natl Cancer Inst 65, 43-46.

[27] McLay RN, Maki PM, Lyketsos CG (2003) Nulliparity and late menopause are associated with decreased cognitive decline. J Neuropsychiatry Clin Neurosci 15, 161-167.

[28] Beeri MS, Rapp M, Schmeidler J, Reichenberg A, Purohit DP, Perl DP, Grossman HT, Prohovnik I, Haroutunian V, Silverman JM (2009) Number of children is associated with neuropathology of Alzheimer's disease in women. Neurobiol Aging 30, 1184-1191.

[29] Sobow T, Kloszewska I (2004) Parity, number of pregnancies, and the age of onset of Alzheimer's disease. J Neuropsychiatry Clin Neurosci 16, 120-121.

[30] Ptok U, Barkow K, Heun R (2002) Fertility and number of children in patients with Alzheimer's disease. Arch Womens Ment Health 5, 83-86.

[31] Hankinson SE, Colditz GA, Hunter DJ, Manson JE, Willett WC, Stampfer MJ, Longcope C, Speizer FE (1995) Reproductive factors and family history of breast cancer in relation to plasma estrogen and prolactin levels in postmenopausal women in the Nurses' Health Study (United States). Cancer Causes Control 6, 217-224.

[32] Davey DA (2013) Alzheimer's disease, dementia, mild cognitive impairment and the menopause: A 'window of opportunity'? Womens Health (Lond Engl) 9, 279-290.

[33] Liu R, Baird D, Park Y, Freedman ND, Huang X, Hollenbeck A, Blair A, Chen H (2014) Female reproductive factors, menopausal hormone use, and Parkinson's disease. Mov Disord 29, 889-896.

[34] Tierney MC, Ryan J, Ancelin ML, Moineddin R, Rankin S, Yao C, MacLusky NJ (2013) Lifelong estrogen exposure and memory in older postmenopausal women. J Alzheimers Dis 34, 601-608.
[35] DeBattista C, Belanoff J (2005) C-1073 (mifepristone) in the adjunctive treatment of Alzheimer's disease. Curr Alzheimer Res 2, 125-129.

[36] Pomara N, Doraiswamy PM, Tun H, Ferris S (2002) Mifepristone (RU 486) for Alzheimer's disease. Neurolog 58, 1436.

[37] Belanoff JK, Jurik J, Schatzberg LD, DeBattista C, Schatzberg AF (2002) Slowing the progression of cognitive decline in Alzheimer's disease using mifepristone. J Mol Neurosci 19, 201-206.

[38] United Nations, Department of Economic and Social Affairs, Population Division (2012) World Contraceptive Use 2012 (POP/DB/CP/Rev2012)

[39] The ESHRE, Capri Workshop, Group (2008) Intrauterine devices and intrauterine systems. Hum Reprod Update 14, 197-208.

[40] Cao ZY (1999) Chinese Obstetrics and Gynecology, People's Medical Publishing House, Beijing.

[41] Niu EM (1985) Endometrial estrogen and progesterone receptors in women wearing intrauterine contraceptive devices. Zhonghua Fu Chan Ke Za Zhi 20, 160-163, 190.

[42] Fahmy K, El-Gazar A, Eisa I, Ghonaim M, Saad S, Afifi A (1991) Levels of serum steroid hormones in intrauterine contraceptive device users. Gynecol Endocrinol 5, 1-5.

[43] Brenner PF, Mishell DJ (1975) Progesterone and estradiol patterns in women using an intrauterine contraceptive device. Obstet Gynecol 46, 456-459.

[44] Hagenfeldt K, Landgren BM (1975) Contraception by intrauterine release of progesterone-effects on endometrial trace elements, enzymes and steroids. J Steroid Biochem $\mathbf{6}$, 895-898.

[45] Faundes A, Segal SJ, Adejuwon CA, Brache V, Leon P, Alvarez-Sanchez F (1980) The menstrual cycle in women using an intrauterine device. Fertil Steril 34, 427-430.

[46] Nygren KG, Johansson ED (1973) Premature onset of menstrual bleeding during ovulatory cycles in women with an intrauterine contraceptive device. Am J Obstet Gynecol 117, 971-975.

[47] de Castro A, Gonzalez-Gancedo P, Contreras F, Lapena G (1986) The effect of copper ions in vivo on specific hormonal endometrial receptors. Adv Contracept 2, 399-404.

[48] Punnonen R, Pettersson K, Vanharanta R (1984) Androgen, estrogen and progestin cytosol receptor concentrations in the normal human endometrium. Effects of intrauterine device. Gynecol Obstet Invest 17, 73-77.

[49] Guleria K, Agarwal N, Mishra K, Gulati R, Mehendiratta A (2004) Evaluation of endometrial steroid receptors and cell mitotic activity in women using copper intrauterine device: Can $\mathrm{Cu}-\mathrm{T}$ prevent endometrial cancer? J Obstet Gynaecol Res 30, 181-187.

[50] Oster G, Salgo MP (1975) The copper intrauterine device and its mode of action. $N$ Engl J Med 293, 432-438.

[51] Bean JA, Leeper JD, Wallace RB, Sherman BM, Jagger H (1979) Variations in the reporting of menstrual histories. Am J Epidemiol 109, 181-185. 